\title{
Emergent high-spin state above 7 GPa in superconducting FeSe
}

\author{
B. W. Lebert,,${ }^{1,2,{ }^{*}}$ V. Balédent,${ }^{3}$ P. Toulemonde, ${ }^{4}$ J. M. Ablett, ${ }^{2}$ and J.-P. Rueff ${ }^{2,5}$ \\ ${ }^{1}$ Sorbonne Université, CNRS, IRD, MNHN, Institut de Minéralogie de Physique des Matériaux et de Cosmochimie, IMPMC, \\ F-75005 Paris, France \\ ${ }^{2}$ Synchrotron SOLEIL, L'Orme des Merisiers, BP 48 St Aubin, 91192 Gif-sur-Yvette, France \\ ${ }^{3}$ Laboratoire de Physique des Solides, CNRS, Univ. Paris-Sud, Université Paris-Saclay 91405 Orsay cedex, France \\ ${ }^{4}$ Université Grenoble Alpes, CNRS, Grenoble INP, Institut Néel, F-38000 Grenoble, France \\ ${ }^{5}$ Sorbonne Université, CNRS, Laboratoire de Chimie Physique - Matière et Rayonnement, LCPMR, F-75005 Paris, France
}

(Received 20 October 2017; revised manuscript received 22 March 2018; published 4 May 2018)

\begin{abstract}
The local electronic and magnetic properties of superconducting FeSe have been investigated by $K \beta$ x-ray emission and simultaneous x-ray absorption spectroscopy (XAS) at the Fe $K$ edge at high pressure and low temperature. Our results indicate a sluggish decrease of the local Fe spin moment under pressure up to $7 \mathrm{GPa}$, in line with previous reports, followed by a sudden increase at higher pressure. The magnetic surge is preceded by an abrupt change of the Fe local structure as observed by the decrease of the XAS preedge region intensity and corroborated by ab initio simulations. This pressure corresponds to a structural transition from the Cmma form to the denser $\mathrm{Pbnm}$ form with octahedral coordination of iron. Finally, the near-edge region of the XAS spectra shows a change before this transition at $5 \mathrm{GPa}$, corresponding well with the onset pressure of the sudden enhancement of $T_{c}$. Our results emphasize the delicate interplay between structural, magnetic, and superconducting properties in FeSe under pressure.
\end{abstract}

DOI: 10.1103/PhysRevB.97.180503

FeSe is the simplest form of the Fe-based superconductors (FeSC), yet one of the more fascinating members, as seen in its many overlapping phases and their apparent correlation with the superconducting critical temperature $T_{c}$ (Fig. 1). However, in spite of intensive research over the past years, the physics of FeSC remains still poorly understood because of the intricate coupling between structural, magnetic, and electronic degrees of freedom. Below 6-7 GPa, FeSe has a PbO-type tetragonal structure $(P 4 / \mathrm{nmm})$ at ambient temperature which is slightly distorted to an orthorhombic structure ( $\mathrm{Cmma})$ [1] below the structural and magnetic transition temperatures $\left(T_{S}\right.$ and $T_{N}$ ). The low-temperature orthorhombic phase, denoted here as ortho-I, is characterized by nematic order with two distinct origins: below $T_{s}$, it is a second-order structural transition believed to be driven by orbital order [2,3]; while below $T_{N}$, it is a simultaneous first-order magnetostructural transition driven by stripe-type spin fluctuations [4-6]. A small magnetic moment with unknown antiferromagnetic (AFM) order exists below $T_{N}$, as seen with muon spin relaxation ( $\mu \mathrm{SR}$ ) at 0.8-2.4 GPa [7-9] and with nuclear forward scattering until $4 \mathrm{GPa}$ [6]. Transport measurements show that this AFM order exists to at least $6.3 \mathrm{GPa}$ [10]. Above 6-7 GPa, a structural transition is observed [11-14] to a MnP-type orthorhombic phase (Pnma or equivalently Pbnm), denoted here as ortho-II, with the Fe ion site symmetry changing from tetrahedral $\left(T_{d}\right)$ to octahedral $\left(O_{h}\right)$ coordination. This transition was observed with ${ }^{57} \mathrm{Fe}$ Mössbauer spectroscopy above $7 \mathrm{GPa}$ as an additional component in the spectrum; however, no

*Blair.Lebert@gmail.com magnetic hyperfine splitting is observed at $4.2 \mathrm{~K}$ [15]. The superconducting critical temperature in FeSe shows drastic changes with pressure. After an initial increase, $T_{c}$ dips at the onset of AFM order, which is attributed to a reduction in the density of states due to a reconstruction of the Fermi surface $[16,17]$. Increasing the pressure further enhances both the magnetic and superconducting orders, with $T_{N}$ reaching a maximum of $45-55 \mathrm{~K}[8,10]$ at $\sim 4.2 \mathrm{GPa}$ and $T_{c}$ plateauing around $20 \mathrm{~K}$ below this pressure $[10,18]$. Above this pressure, magnetic order decreases and coincides with a sharp rise in $T_{c}$ to a maximum around $37 \mathrm{~K}$ at $\sim 6.2 \mathrm{GPa}[10,12,13,15,19]$. After this maximum, $T_{c}$ has a discontinuous change from a positive to a negative slope, coinciding with the transition to the ortho-II phase [11]. An AFM region in the middle of a superconducting "dome" is quite unique, and the phase diagram shows their intimate connection as two $T_{c}$ anomalies where the two intersect.

This macroscopic complexity reflects the strong coupling between charge, spin, and structure which in turn affects the superconducting properties. It is therefore of great importance to be able to probe these different degrees of freedom at the local level while exploring the phase diagram in the vicinity of the superconducting regions. X-ray spectroscopy in the hard X-ray range is well suited to that aim while being compatible with high-pressure conditions [20]. In particular, $K \beta(3 p \rightarrow 1 s$ transition) $\mathrm{x}$-ray emission spectroscopy (XES) and $\mathrm{x}$-ray absorption spectroscopy (XAS) at the $K$ edge are well-established probes of the local electronic, magnetic, and structural properties. As a primarily atomic probe, XES can access the local magnetic moment of a selected atom regardless of the magnetic order, while XAS provides a view of the local 


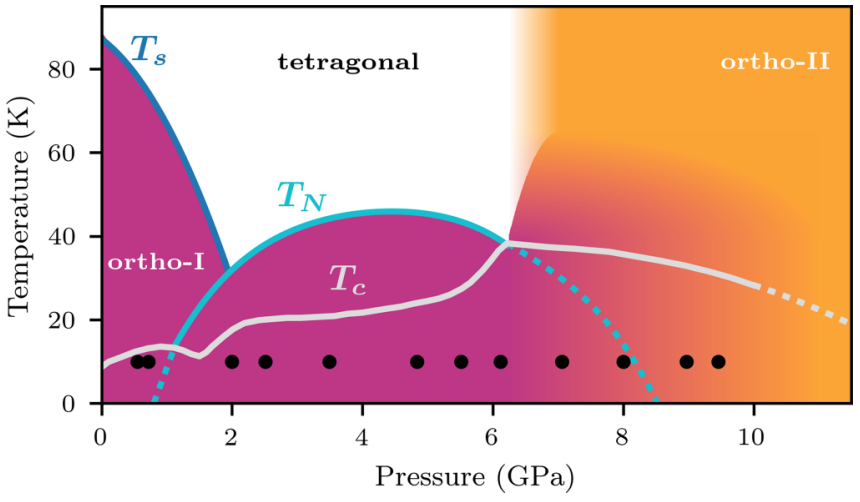

FIG. 1. Schematic $P-T$ phase diagram of FeSe [10,11]. Tetragonal (white), ortho-I (purple), and ortho-II (orange) structures are described in the text. $T_{s}, T_{N}$, and $T_{c}$ are the structural, magnetic, and superconducting transitions, with dashed lines representing extrapolations. $P-T$ measurement points for XES/XAS are indicated with circles.

electronic and structural properties. In recent works [21,22], Kumar et al. and Chen et al. have used XES to probe Fe magnetism in FeSe under high pressure. Their results show a decrease of the $\mathrm{Fe}$ spin state with a discontinuous change in slope near the ortho-I $\rightarrow$ ortho-II transition. This is interpreted as a high-spin (HS) to low-spin (LS) transition [21] or a state with a smaller magnetic moment [22]. Their experiments were performed with polycrystalline samples including the hexagonal polymorph of $\mathrm{FeSe}(11 \%$ in the sample of Chen et al.) and at room temperature or only up to $8 \mathrm{GPa}$ at $8 \mathrm{~K}$ for Kumar et al. In this work, we perform a high-pressure study of FeSe single crystals at $10 \mathrm{~K}$ until $9.5 \mathrm{GPa}$ using Fe- $K \beta$ XES and XAS at the Fe $K$ edge. Our results demonstrate a LS $\rightarrow$ HS transition in the same pressure region as the ortho-I $\rightarrow$ ortho-II transition and the maximum of $T_{c}$. The spin state transition coincides with the structural transition and $T_{c}$ slope change thus demonstrating the strong interplay between electronic and lattice degrees of freedom, adding to the remarkable properties of FeSe, and more generally to Fe superconductors. Here, we report a LS to HS transition under pressure in a $3 d$ metal compound which challenges the classical crystal-field derived picture of spin transitions in these materials. We assign the low-spin state here to the atypical orbital-selective "Mottness" of the $3 d$ electrons in $\mathrm{Fe}$ superconductors.

The XES/XAS experiment was performed on the GALAXIES beamline [23] at the SOLEIL synchrotron facility. We used high-purity FeSe single crystals from the Institut Néel (Grenoble) grown by chemical vapor transport [24]. Pressure was applied using a membrane-driven diamond-anvil cell equipped with 1.2 -mm-thick diamonds with $300-\mu \mathrm{m}$ culets. Several FeSe single crystals were loaded in a $150-\mu \mathrm{m}$ hole of a $\mathrm{CuBe}$ gasket, along with ruby chips for in situ pressure measurement [25] and silicone oil as a pressure transmitting medium. XES/XAS were measured with the spectrometer in a transmission geometry using a 1-m-radius spherically bent $\mathrm{Ge}(620)$ crystal analyzer and an avalanche photodiode detector arranged in the Rowland circle geometry. The total energy resolution at the $\mathrm{Fe} K \beta$ line $\left(\sim 7057 \mathrm{eV}, \theta_{B}=79^{\circ}\right)$ was $1.2 \mathrm{eV}$ FWHM. The XES spectra were measured with $10 \mathrm{-keV}$

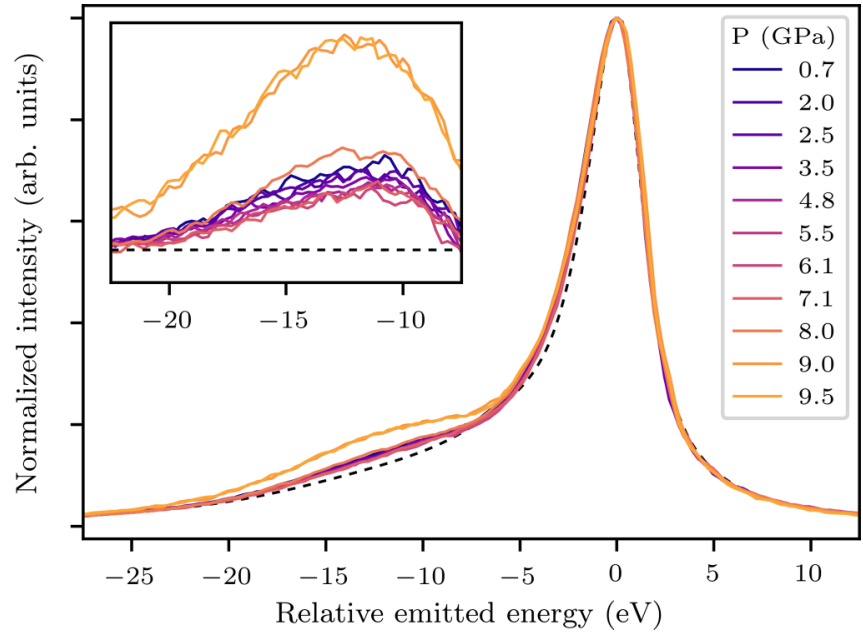

FIG. 2. Fe $K \beta$ x-ray emission in FeSe as a function of pressure measured at $10 \mathrm{~K}$. The inset is a zoom of the satellite region showing the difference with the $\mathrm{FeS}_{2}$ zero-spin reference [26], shown as a black dashed line in the main plot.

incident $\mathrm{x}$ rays. The XAS spectra was measured using the partial-fluorescence yield (PFY) method with the spectrometer fixed to the $\mathrm{Fe} K \beta$ line. This technique leads to an intrinsic sharpening effect due to the shallower $3 p$ core hole left in the final state with respect to the deeper $1 s$ level [20]. The energies of our XAS spectra have been calibrated using the low-pressure XAS spectrum of FeSe from Chen et al. as a reference.

Our XES spectra measured at $10 \mathrm{~K}$ are shown in Fig. 2 for increasing pressure up to $9.5 \mathrm{GPa}$. The spectra are aligned to the main peak at $7057 \mathrm{eV}$ and normalized to the maximum intensity. There is a weak satellite located around $-12 \mathrm{eV}$ from the main line, which is well established as a signature of the local magnetic moment [27]. The satellite can be seen more clearly by subtracting the zero-spin reference [26], $\mathrm{FeS}_{2}$, as shown in the inset of Fig. 2. The fitted intensity of the satellite from the difference spectra is shown in Fig. 3 as red squares. FeSe exhibits a LS state with a gradually decreasing magnetic moment until 6-7 GPa. The magnetic moment starts to increase between 7 and $8 \mathrm{GPa}$ and then jumps between 8 and $9 \mathrm{GPa}$ to a HS state which plateaus around $9.5 \mathrm{GPa}$. The low-pressure behavior is consistent with previous results $[21,22]$ and is understood to be due to band-structure effects in the compressed lattice; however, the sudden increase around $8 \mathrm{GPa}$ was previously unobserved. The pressure region of the transformation of this LS $\rightarrow$ HS transition coincides with the ortho-I $\rightarrow$ ortho-II coexistence region at low temperature [11].

To gain insight into the $\mathrm{Fe}$ local properties, we also measured high-resolution XAS at each pressure point after each XES measurement. The series of PFY-XAS spectra taken at the same pressures as XES is shown in Fig. 4, where the inset emphasizes the preedge region which is sensitive to the $3 d$ states. The spectra are normalized and flattened using the software ATHENA [28]. Subsequently, the postedge points are binned to increase statistics. The prepeak feature A in FeSe at low pressure is mainly due to the dipole transitions from $\mathrm{Fe} 1 s$ to $\mathrm{Fe} 3 d$-Se $4 p$ hybrid bands as expected in $T_{d}$ symmetry, with a smaller contribution from $\mathrm{Fe}$ quadrupole 


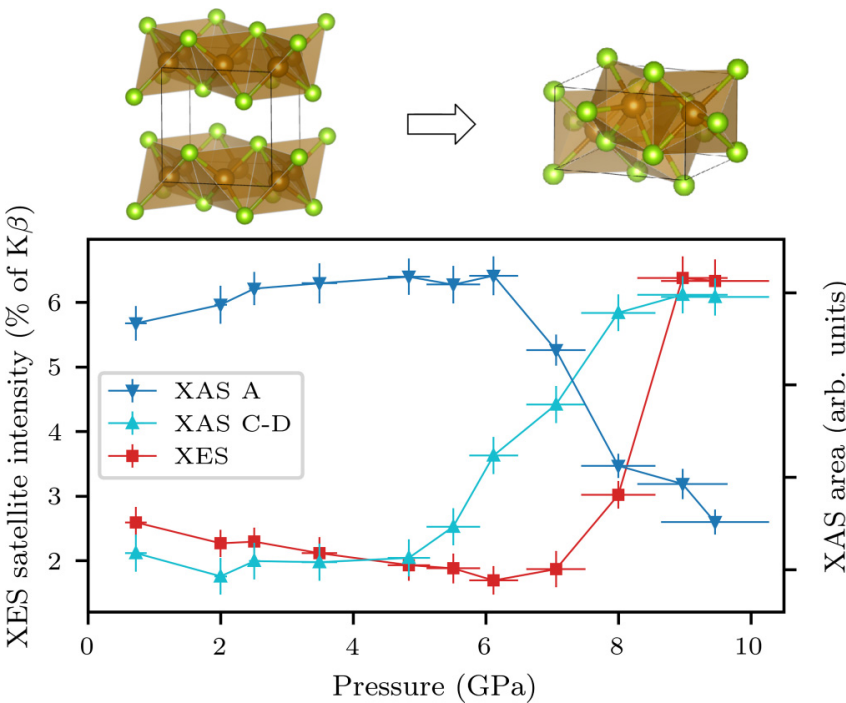

FIG. 3. Top: Schematic of ortho-I to ortho-II structural transition. Bottom: Pressure dependence of XES and XAS spectra at $10 \mathrm{~K}$. Left scale: Intensity of $K \beta$ satellite shown as red squares. Right scale: Area of the Fe preedge feature A shown as blue inverted triangles and area between XAS features C and D shown as cyan triangles (scaled and offset).

$1 s \rightarrow 3 d$ transitions [22,29-31]. Fe $3 d$-Se $4 p$ hybridization is sensitive to the local geometry, therefore the prepeak can be used primarily to study the pressure evolution of the structure (and the spin state as discussed below). To clarify its pressure dependence, it was fitted by a Gaussian line shape after subtraction of a background (error function + linear) to account for the rising edge. This preedge area is shown as blue inverted triangles in Fig. 3 using the right scale and the label "XAS A." As FeSe is compressed the prepeak area slowly increases until $6 \mathrm{GPa}$, above which it decreases with a much sharper slope until our highest pressure point at $9.5 \mathrm{GPa}$. The decreased intensity is due to a reduced hybridization as the

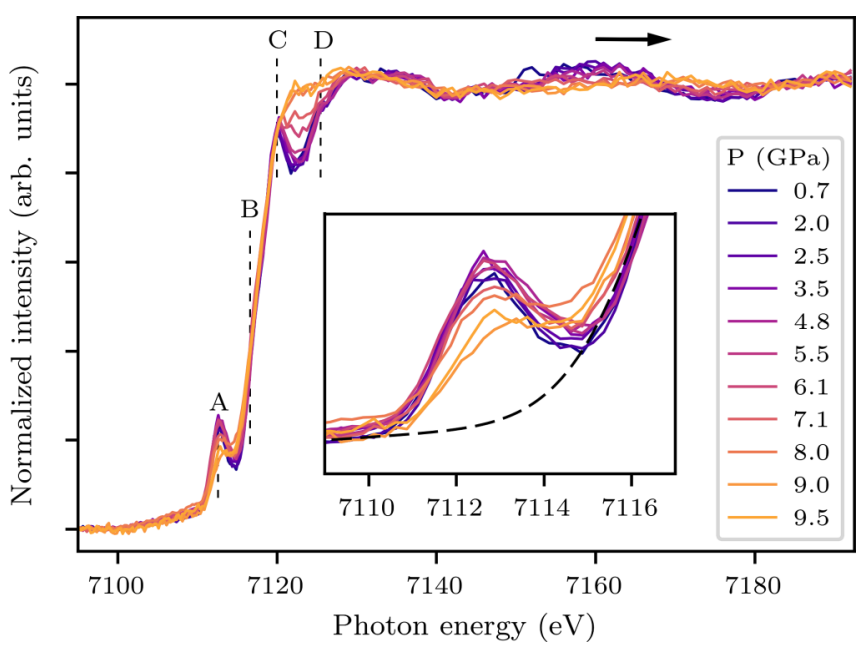

FIG. 4. Partial-fluorescence yield x-ray absorption at the Fe $K$ edge in $\mathrm{FeSe}$ as a function of pressure measured at $10 \mathrm{~K}$. The inset is a zoom of the preedge region, with the $0 \mathrm{GPa}$ background (error function + linear) shown with a dashed line.
Fe-Se bond lengths increase by $\approx 5 \%[13]$ and the coordination of iron increases during the ortho-I $\left(T_{d}\right) \rightarrow$ ortho-II $\left(O_{h}\right)$ transition [11]. The increased coordination is also supported by the change of the hyperfine splitting at high pressure [15]. The remarkable parallel evolution of the magnetic (XES) and structural (XAS) properties shows the interplay between the electronic and lattice degrees of freedom. The fact that the structural transition precedes the magnetic transition suggests that it is not magnetically driven.

The absorption features B, C, and D can be assigned as Fe dipole $1 s \rightarrow 4 p$ transitions, where $\mathrm{C}$ and $\mathrm{D}$ also have significant contribution from $\mathrm{Fe} 1 s$ to $\mathrm{Fe} 4 p$-Se $3 d$ hybrid band transitions [22,29-31]. The features at higher energies are dominated by multiple scattering of the photoelectron with the nearest neighbors. The feature around $7160 \mathrm{eV}$ shows a clear shift (arrow in Fig. 4) supporting the change of local structure we see with the prepeak. A significant spectral change is observed in the region between features $C$ and $D$, even before the structural and magnetic transitions. The change is shown in Fig. 3 (cyan triangles) by taking the area between $C$ and $D$. The onset of the increased intensity corresponds remarkably well with the sudden increase in $T_{c}$ around $5 \mathrm{GPa}$ [10]. The trend is continuous until the ortho-II dominant phase at $9.5 \mathrm{GPa}$, which puts into question the possible connection between ortho-II and superconductivity since ortho-II is not believed to support superconductivity [11], and a negative $d \rho / d \mathrm{~T}$ suggests it is a semiconductor $[10,15]$.

To further understand the structural and electronic transitions, the XAS spectra were simulated using the FDMNES code [32]. A cluster radius of $12 \AA$ (10 $\AA$ ) for the low pressure (high pressure) reached full convergence. Spin-orbit coupling was included by relativistic effect corrections and the Fermi level was set self-consistently [33]. The crystal structures were taken from Ref. [1] for the low-pressure ortho-I phase and from Ref. [14] for the high-pressure ortho-II phase. To simulate the pressure-induced spin-state transition, the calculations in both phases were carried out using either a LS $\left(3 d_{\uparrow}^{3} 3 d_{\downarrow}^{3}\right)$ or a HS $\left(3 d_{\uparrow}^{5} 3 d_{\downarrow}^{1}\right)$ ground-state configuration.

The results are shown in Fig. 5 along with the experimental spectra measured at 0.7 and $9.0 \mathrm{GPa}$. We do not expect to yield an accurate description of the $d$ electronic structure in FeSe without including correlations-more accurate approaches show that many-body effects lead to a renormalization of the $d$ bands and changes of the $d$ density of states close to the Fermi edge [34] - but the $K$ edge should be well described since it mostly connects to a single-particle picture. We find that the calculations for both the low-pressure and high-pressure phases compare well to the experimental spectra in the preedge and near-edge regions. We notice, however, that the intensity of the preedge for the high-pressure phase is overestimated. The preedge intensity is strongly dependent on minor distortions of the metal site which favor or disfavor dipolar transitions to ligand $p$ states in addition to the weaker quadrupolar transition to the $d$ states. On the contrary, the preedge shape is mostly related to the orbitals' energy splitting and spin state, therefore it is a much better fingerprint of the local $3 d$ electronic properties. The preedge shape matches very well with a high-spin (low-spin) configuration for the high-pressure (low-pressure) phase. The less-pronounced prepeak in ortho-II 


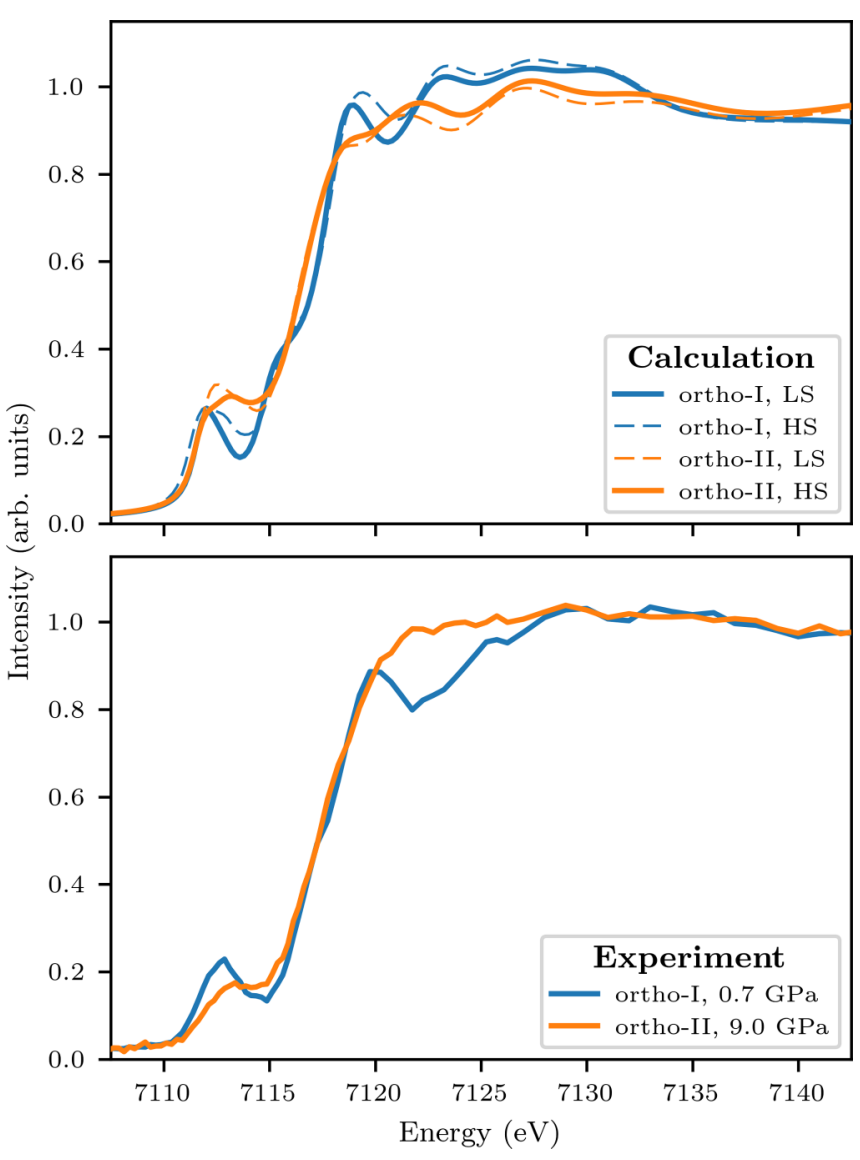

FIG. 5. Comparison of calculated and measured XAS spectra at the Fe $K$ edge in FeSe. Top panel: Calculated XAS spectra for the ortho-I phase (Cmma) and the ortho-II phase (Pnma) in low-spin (LS) and high-spin (HS) configurations. Thick lines represent the best agreement with experiment. Bottom panel: Experimental XAS spectra measured at $10 \mathrm{~K}$.

is consistent with high-spin $\mathrm{Fe}^{\mathrm{II}}$ in an octahedral environment [35]. This agrees with our XES results that there is a transition to a high-spin state above $7 \mathrm{GPa}$ at $10 \mathrm{~K}$ in FeSe.
The main outcome of our study is the original observation of a high-spin state above $7 \mathrm{GPa}$ as FeSe adopts the ortho-II phase. This follows the initial decrease of the local moment in the ortho-I phase which has been reported elsewhere [21,22] and confirmed here. Both XAS and XES indicate that the magnetic surge at high pressure is consistent with a pressure-induced LS to HS transition. The electronic change is likely resulting from the change of Fe site symmetry: in the ortho-I phase, Fe occupies a tetrahedral $\left(T_{d}\right)$ site that becomes increasingly distorted with pressure, whereas Fe sits in an octahedral $\left(O_{h}\right)$ site in the ortho-II phase [11-13]. At first glance, the spin state of FeSe is surprising. According to ligand field theory, one would expect $\mathrm{Fe}$ to be high spin in the ortho-I phase since tetrahedral coordination normally favors a high-spin state. However, the Fe-based superconductor's electronic structure was also shown to be controlled by the correlated and specific Mottness of $d$ electrons with band-dependent correlations yielding $e_{g}$ states less correlated than $t_{2 g}$ states [36]. We suggest that the $T_{d}$ low-spin state is stabilized because it minimizes the on-site electron correlations by filling preferentially the $e_{g}$ bands. At high pressure, correlation becomes less effective as the bandwidth broadens which eventually allows a high-spin configuration as the Fe symmetry turns $O_{h}$. In line with recent band-structure calculations [37], we can further argue that the exceptional stability of the magnetism at high pressure in FeSe could be aided by the increased three-dimensional character of the compressed lattice and change of the Fermi surface.

We acknowledge SOLEIL for the provision of synchrotron radiation facilities (Proposal No. 20151119) and help from the high-pressure laboratory for cell loading. We acknowledge P. Strobel and S. Karlsson from Institut Néel, Grenoble, France, for their help in the growth of the FeSe crystals using the chemical vapor transport method. B.W.L. acknowledges financial support from the French state funds managed by the ANR within the "Investissements d'Avenir" programme under reference ANR-11-IDEX-0004-02, and within the framework of the Cluster of Excellence MATISSE led by Sorbonne Université and from the LLB/SOLEIL PhD fellowship programme.
[1] S. Margadonna, Y. Takabayashi, M. T. McDonald, K. Kasperkiewicz, Y. Mizuguchi, Y. Takano, A. N. Fitch, E. Suard, and K. Prassides, Chem. Commun., 5607 (2008).

[2] S.-H. Baek, D. V. Efremov, J. M. Ok, J. S. Kim, J. van den Brink, and B. Büchner, Nat. Mater. 14, 210 (2015).

[3] P. Massat, D. Farina, I. Paul, S. Karlsson, P. Strobel, P. Toulemonde, M.-A. Méasson, M. Cazayous, A. Sacuto, S. Kasahara, T. Shibauchi, Y. Matsuda, and Y. Gallais, Proc. Natl. Acad. Sci. USA 113, 9177 (2016).

[4] Q. Wang, Y. Shen, B. Pan, Y. Hao, M. Ma, F. Zhou, P. Steffens, K. Schmalzl, T. R. Forrest, M. Abdel-Hafiez, X. Chen, D. A. Chareev, A. N. Vasiliev, P. Bourges, Y. Sidis, H. Cao, and J. Zhao, Nat. Mater. 15, 159 (2016).

[5] P. S. Wang, S. S. Sun, Y. Cui, W. H. Song, T. R. Li, R. Yu, H. Lei, and W. Yu, Phys. Rev. Lett. 117, 237001 (2016).

[6] K. Kothapalli, A. E. Böhmer, W. T. Jayasekara, B. G. Ueland, P. Das, A. Sapkota, V. Taufour, Y. Xiao, E. Alp, S. L. Bud'ko, P.
C. Canfield, A. Kreyssig, and A. I. Goldman, Nat. Commun. 7, 12728 (2016).

[7] M. Bendele, A. Amato, K. Conder, M. Elender, H. Keller, H.-H. Klauss, H. Luetkens, E. Pomjakushina, A. Raselli, and R. Khasanov, Phys. Rev. Lett. 104, 087003 (2010).

[8] M. Bendele, A. Ichsanow, Y. Pashkevich, L. Keller, T. Strässle, A. Gusev, E. Pomjakushina, K. Conder, R. Khasanov, and H. Keller, Phys. Rev. B 85, 064517 (2012).

[9] R. Khasanov, Z. Guguchia, A. Amato, E. Morenzoni, X. Dong, F. Zhou, and Z. Zhao, Phys. Rev. B 95, 180504 (2017).

[10] J. P. Sun, K. Matsuura, G. Z. Ye, Y. Mizukami, M. Shimozawa, K. Matsubayashi, M. Yamashita, T. Watashige, S. Kasahara, Y. Matsuda, J. Q. Yan, B. C. Sales, Y. Uwatoko, J. G. Cheng, and T. Shibauchi, Nat. Commun. 7, 12146 (2016).

[11] V. Svitlyk, M. Raba, V. Dmitriev, P. Rodière, P. Toulemonde, D. Chernyshov, and M. Mezouar, Phys. Rev. B 96, 014520 (2017). 
[12] G. Garbarino, A. Sow, P. Lejay, A. Sulpice, P. Toulemonde, M. Mezouar, and M. Nunez-Regueiro, Europhys. Lett. 86, 27001 (2009).

[13] S. Margadonna, Y. Takabayashi, Y. Ohishi, Y. Mizuguchi, Y. Takano, T. Kagayama, T. Nakagawa, M. Takata, and K. Prassides, Phys. Rev. B 80, 064506 (2009).

[14] R. S. Kumar, Y. Zhang, S. Sinogeikin, Y. Xiao, S. Kumar, P. Chow, A. L. Cornelius, and C. Chen, J. Phys. Chem. B 114, 12597 (2010).

[15] S. Medvedev, T. M. McQueen, I. A. Troyan, T. Palasyuk, M. I. Eremets, R. J. Cava, S. Naghavi, F. Casper, V. Ksenofontov, G. Wortmann, and C. Felser, Nat. Mater. 8, 630 (2009).

[16] T. Terashima, N. Kikugawa, A. Kiswandhi, D. Graf, E.-S. Choi, J. S. Brooks, S. Kasahara, T. Watashige, Y. Matsuda, T. Shibauchi, T. Wolf, A. E. Böhmer, F. Hardy, C. Meingast, H. v. Löhneysen, and S. Uji, Phys. Rev. B 93, 094505 (2016).

[17] T. Terashima, N. Kikugawa, S. Kasahara, T. Watashige, Y. Matsuda, T. Shibauchi, and S. Uji, Phys. Rev. B 93, 180503 (2016).

[18] K. Miyoshi, K. Morishita, E. Mutou, M. Kondo, O. Seida, K. Fujiwara, J. Takeuchi, and S. Nishigori, J. Phys. Soc. Jpn. 83, 013702 (2014).

[19] D. Braithwaite, B. Salce, G. Lapertot, F. Bourdarot, C. Marin, D. Aoki, and M. Hanfland, J. Phys.: Condens. Matter 21, 232202 (2009).

[20] J.-P. Rueff and A. Shukla, Rev. Mod. Phys. 82, 847 (2010).

[21] R. S. Kumar, Y. Zhang, Y. Xiao, J. Baker, A. Cornelius, S. Veeramalai, P. Chow, C. Chen, and Y. Zhao, Appl. Phys. Lett. 99, 061913 (2011).

[22] J. M. Chen, S. C. Haw, J. M. Lee, T. L. Chou, S. A. Chen, K. T. Lu, Y. C. Liang, Y. C. Lee, N. Hiraoka, H. Ishii, K. D. Tsuei, E. Huang, and T. J. Yang, Phys. Rev. B 84, 125117 (2011).

[23] J.-P. Rueff, J. M. Ablett, D. Céolin, D. Prieur, T. Moreno, V. Balédent, B. Lassalle, J. E. Rault, M. Simon, and A. Shukla, J. Synchrotron Radiat. 22, 175 (2015).
[24] S. Karlsson, P. Strobel, A. Sulpice, C. Marcenat, M. Legendre, F. Gay, S. Pairis, O. Leynaud, and P. Toulemonde, Supercond. Sci. Technol. 28, 105009 (2015).

[25] A. Dewaele, M. Torrent, P. Loubeyre, and M. Mezouar, Phys. Rev. B 78, 104102 (2008).

[26] J. F. Lin, H. Watson, G. Vankó, E. E. Alp, V. B. Prakapenka, P. Dera, V. V. Struzhkin, A. Kubo, J. Y. Zhao, C. McCammon, and W. J. Evans, Nat. Geosci. 1, 688 (2008).

[27] G. Peng, F. M. F. de Groot, K. Hämäläinen, J. A. Moore, X. Wang, M. M. Grush, J. B. Hastings, D. P. Siddons, W. H. Amstrong, O. C. Mullins, and S. P. Cramer, J. Am. Chem. Soc. 116, 2914 (1994).

[28] B. Ravel and M. Newville, J. Synchrotron Radiat. 12, 537 (2005).

[29] J. M. Chen, S. C. Haw, J. M. Lee, S. A. Chen, K. T. Lu, M. J. Deng, S. W. Chen, H. Ishii, N. Hiraoka, and K. D. Tsuei, J. Chem. Phys 137, 244702 (2012).

[30] M. Bendele, C. Marini, B. Joseph, L. Simonelli, P. Dore, S. Pascarelli, M. Chikovani, E. Pomjakushina, K. Conder, N. L. Saini, and P. Postorino, J. Phys.: Condens. Matter 25, 425704 (2013).

[31] B. Joseph, A. Iadecola, L. Simonelli, Y. Mizuguchi, Y. Takano, T. Mizokawa, and N. L. Saini, J. Phys.: Condens. Matter 22, 485702 (2010).

[32] S. A. Guda, A. A. Guda, M. A. Soldatov, K. A. Lomachenko, A. L. Bugaev, C. Lamberti, W. Gawelda, C. Bressler, G. Smolentsev, A. V. Soldatov, and Y. Joly, J. Chem. Theory Comput. 11, 4512 (2015).

[33] O. Bunău and Y. Joly, J. Phys.: Condens. Matter 21, 345501 (2009).

[34] M. Aichhorn, S. Biermann, T. Miyake, A. Georges, and M. Imada, Phys. Rev. B 82, 064504 (2010).

[35] T. E. Westre, P. Kennepohl, J. G. DeWitt, B. Hedman, K. O. Hodgson, and E. I. Solomon, J. Am. Chem. Soc. 119, 6297 (1997).

[36] L. de' Medici, G. Giovannetti, and M. Capone, Phys. Rev. Lett. 112, 177001 (2014).

[37] D. S. Parker, Sci. Rep. 7, 3388 (2017). 